\title{
HRJ
}

V.3 n.15 (2022)

Recebido: 16/12/2021

Aceito: 12/01/2022

\section{Mucormicose associada à COVID-19: análise com base em revisão sistemática da literatura}

\author{
Juliana Maria Nascimento Silva ${ }^{1}$ \\ Carlos Augusto Almeida Correa ${ }^{2}$
}

\footnotetext{
${ }^{1}$ Residente em Clínica Médica pelo Programa de Residência Médica pela Fundação de Ensino e Pesquisa em Ciências da Saúde - FEPECS/SES-DF. Lattes: http://lattes.cnpq.br/8464624622554903

${ }^{2}$ Médico da Secretaria de Saúde do Distrito Federal, Preceptor do Programa de Residência Médica pela Fundação de Ensino e Pesquisa em Ciências da Saúde - FEPECS/SES-DF. Especialista em Clínica Médica e Terapia Intensiva. Lattes: http://lattes.cnpq.br/9635585359695907
}

\section{RESUMO}

Introdução: Com a pandemia da COVID-19 surgiu a associação de algumas doenças oportunistas como a mucormicose, uma infecção fúngica que acomete os sistemas rinocerebral e gástrico. Apesar da maior prevalência de casos na Índia, o Brasil também apresentou relatos de casos. O objetivo desta revisão foi trazer um levantamento atualizado dos casos descritos. Métodos: Trata-se de uma revisão integrativa, de estudos disponibilizados nas bases de dados Scielo, Pubmed e Medline. Resultados: A partir dos estudos avaliados foram observadas características predominantes nos pacientes COVID-19 positivo associados à mucormicose: homens, diabéticos e hipertensos, em tratamento com corticosteroides. O possível mecanismo de associação nesses pacientes deve-se a hiperglicemia que aumenta a tempestade de citocinas, já presente devido a infecção por SarsCov-2, levando a destruição endotelial e comprometimento dos órgãos, além de alterações no metabolismo do íon ferro, resultando em alta taxa de mortalidade desses indivíduos. Conclusões: Considerando a complexidade desses pacientes, é importante o acompanhamento e monitoramento dos mesmo para melhor compreensão dos mecanismos e impactos dessa associação.

Palavras-chave: Mucormicose, COVID-19, Infecções oportunistas.

\section{COVID-19-associated mucormycosis: analysis based on systematic literature review}

\begin{abstract}
Introduction: The COVID-19 pandemic came the association of some opportunistic diseases such as mucormycosis, a fungal infection that affects the rhinocerebral and gastric systems. Despite the higher prevalence of cases in India, Brazil also presented case reports. The aim of this review was to provide an updated survey of the cases described. Methods: This is an integrative review of studies available in the Scielo, Pubmed and Medline databases. Results: From the studies evaluated predominant characteristics were observed in COVID-19-positive patients associated with mucormycosis: men, diabetics and hypertensive, under treatment with corticosteroids. The possible mechanism of association in these patients is due to hyperglycemia that increases the cytokine storm, already present due to Sars-Cov-2 infection, leading to endothelial destruction and organ involvement, in addition to changes in iron ion metabolism, resulting in high mortality rate of these individuals. Conclusion: Considering the
\end{abstract}


complexity of these patients, it is important to follow up and monitor them for a better understanding of the mechanisms and impacts of this association.

Keywords: Mucormycosis, COVID-19, Opportunistic diseases.

\section{INTRODUÇÃO}

A pandemia provocada pelo SARS-CoV-2 desde o seu início tem alertado a comunidade de saúde sobre algumas infecções oportunistas. Globalmente vários casos de mucormicose associada à COVID-19 foram relatados ${ }^{1-2}$.

Devido ao crescimento de casos de COVID-19 na Índia, foram documentados cerca de 15 mil casos de mucormicose associados a essa doença ${ }^{1,3}$, o que levou o governo indiano declarar como pandemia o surto dessa infecção fúngica ${ }^{4}$.

Na América do Sul, alguns países como Uruguai e Paraguai evidenciaram alguns casos, e no Brasil houve registros da infecção nos estados do Amazonas, São Paulo, Mato Grosso do Sul e Santa Catarina ${ }^{1}$. Destaca-se que no Brasil, não é esperado a mesma proporção de casos de mucormicose associado à COVID-19 quando comparado à Índia pois este registra elevada incidência de mucormicose quando comparado a outros países do mundo ${ }^{1}$.

Por ser um vírus relativamente novo, os sinais e sintomas indicativos da COVID-19 ainda são insuficientes. Apesar de haver uma relação causal entre mucormicose e COVID-19, alguns fatores como uso de glicocorticoides, diabetes mellitus, uso de corticosteroides sistêmicos a longo prazo, alterações no metabolismo do ferro, linfopenia induzida por vírus foram relacionados ao desenvolvimento da mucormicose em pacientes com COVID-19 $9^{2,5-9}$.

Dessa forma, devido a disponibilidade de informações sobre a relação da mucormicose em pacientes com COVID-19 ser complexa em função das características do paciente e desfecho clínico, o objetivo desta revisão foi trazer um levantamento atualizado dos casos descritos de pacientes com mucormicose associados à COVID-19. 


\section{METODOLOGIA}

O presente trabalho trata-se de uma revisão integrativa que se baseia na definição de uma análise sobre um tema que já foi estudado e discutido em pesquisas anteriores. Essa metodologia consiste em seis etapas, onde a primeira é a definição da pergunta do estudo, a segunda a seleção dos artigos científicos a serem revisados, a terceira a categorização e a avaliação desses estudos. As últimas etapas do processo são a interpretação dos resultados e apresentação da síntese do conhecimento desses trabalhos.

O período do levantamento dos dados foi realizado nos meses de setembro a novembro de 2021. As bases de dados pesquisadas foram Pubmed, Scielo, Medline e protocolos em sites oficiais do governo brasileiro por meio das seguintes palavras-chave: mucormicose (mucormycosis); mucormicose e COVID-19 (mucormycosis covid); infecções por coronavírus (coronavírus infection).

\section{Critérios de inclusão}

- Abordar a presença de mucormicose em pacientes com COVID-19 positivo.

- Publicações indexados nas bases de dados citadas, que fossem publicados em português, inglês ou espanhol.

- Foram consideradas publicações do último ano (2020 a 2021).

- Estivessem disponíveis na íntegra.

- Foram escolhidos somente artigos completos originais e revisões integrativas ou sistemáticas.

\section{Critérios de exclusão}

- Foram excluídos todos os artigos de acesso restrito.

- Foram excluídas publicações anteriores ao ano de 2020. 


\section{RESULTADOS E DISCUSSÃO}

A mucormicose é uma doença causada por fungos da família Mucorales, dos gêneros Rhizopus spp., Rhizomucor spp. ou Mucor spp., Lichtheimia spp, Cunninghamella spp. É considerada uma doença fúngica oportunista, descrita pela primeira vez pelo patologista alemão Richard Paultauf, em 1885, relacionando essa infecção sistêmica com envolvimento gástrico e rinocerebral ${ }^{3}$.

As principais formas de contaminação para o desenvolvimento da mucormicose se dá através da inalação de esporos por fontes ambientais; por via cutânea ou mucosa, quando há ruptura da barreira por ferida, traumas ou queimaduras; ou por via digestiva através de produtos contaminados ${ }^{3}$. Em pacientes que apresentam COVID-19, quando há lesão pulmonar e imunossupressão, o fungo pode disseminar-se nas vias aéreas e seios paranasais e, para outros tecidos à distância.

A mucormicose causa a maioria das suas infecções no nariz e nos seios paranasais, porém existem a forma pulmonar e rino-órbito-cerebral da doença (ROC). Essa última pode se estender para os olhos ou cérebro, causando perda da visão, dor de cabeça e convulsões ${ }^{10}$.

Pacientes diabéticos são propensos a desenvolver a forma ROC, enquanto pacientes imunodeprimidos neutropênicos e transplantados tendem a desenvolver a forma pulmonar ${ }^{1}$. Em pacientes com COVID-19 que apresentam associação com a mucormicose, denominou-se COVID-19 associated mucormycosis - $\mathrm{CAM}^{4}$.

Um dos estudos avaliados analisaram 99 casos notificados de mucormicose associados à COVID-19 até o período de maio de 2021. Foi descrito que a maioria dos relatos observados foram na Índia (72\%), com predominância do sexo masculino (78\%), histórico subjacente de diabetes mellitus $(85 \%)$ e tratamento com glicocorticoides para manejo da COVID-19 (85\%). Na maioria dos pacientes que apresentaram mucormicose, a COVID-19 
foi manifestada de forma grave/crítica (67\%), com elevada taxa de mortalidade (34\%), porém também foi desenvolvida a doença em pacientes com a forma leve/moderada $(24 \%)^{2}$.

$\mathrm{Na}$ Índia, entre janeiro e março de 2021 foi realizado um estudo prospectivo ${ }^{11}$, observacional e multi-centro que incluiu 47 pacientes consecutivos com mucormicose, diagnosticados durante o curso da doença COVID-19. A média de idade para os pacientes foi de $55 \pm 12,8$ anos, e a maioria sofria de diabetes mellitus (76,6\%). A maioria não foi vacinada por COVID-19 (66,0\%) e 91,5\% havia desenvolvido pneumonia moderada a grave, enquanto 42,6\% necessitou de ventilação invasiva. Todos os pacientes receberam corticosteroides e antibióticos de amplo espectro, enquanto a maioria $(78,7 \%)$ recebeu pelo menos um medicamento antiviral. Grande parte dos pacientes $(72,7 \%)$ foram a óbito ${ }^{11}$.

Relato de caso na Turquia $^{12}$ descreveu paciente homem, hospitalizado devido a pneumonia grave desenvolvida pela COVID-19. Esse paciente recebeu altas doses de corticosteroides a longo prazo, desenvolvendo mucormicose maxilo-facial e morreu de sepse. Além do relato de caso o grupo fez pesquisa de literatura encontrando 30 publicações nas quais a maioria dos pacientes eram da Índia (68\%) e homens (76\%). Os fatores de risco descritos foram uso de corticosteroides $(90,5 \%)$, diabetes $(79 \%)$ e hipertensão (34\%). Os tipos de mucormicose mais frequentes foram o rino-orbital (50\%), seguido pelo rino-sinusal (17\%) e rino-orbito-cerebral (15\%), com alta taxa de mortalidade entre os pacientes $(33,3 \%)^{12}$.

Uma revisão de literatura ${ }^{8}$ que considerou 19 estudos envolvendo 20 pacientes observaram prevalência masculina dos casos (70\%), com idade média de 52,2 anos. Geograficamente a maioria dos casos foi entre EUA (30\%) e Índia (25\%), dos quais $50 \%$ dos pacientes eram diabéticos e 35\% apresentavam hipertensão. 50\% dos pacientes apresentaram infecção do tipo-orbital e $30 \%$ manifestaram mucormicose pulmonar. $O$ uso de corticosteroides foi relatado em $60 \%$ desses pacientes, com taxa de mortalidade em $65 \%$ dos $\operatorname{casos}^{8}$. 
Visto que a maioria dos pacientes que apresentam CAM são diabéticos, o mecanismo potencial do aumento da morbidade nesses pacientes e sua mortalidade foi proposto com base nas seguintes alterações: a) eliminação viral reduzida; b) diminuição da função das células T; c) tempestade de citocinas; d) imunossupressão ${ }^{13}$. A hiperglicemia agrava a tempestade de citocinas levando ao rompimento das células endoteliais que provocam danos em múltiplos órgãos em pacientes com COVID-19 ${ }^{7}$. Além disso, durante a cetoacidose diabética, devido ao ambiente ácido e ao aumento dos íons ferro livres há predisposição para o crescimento do Mucorales ${ }^{13}$.

A COVID-19 contribui para várias mudanças no organismo do paciente incluindo o metabolismo do ferro. Foi observado elevados níveis de ferritina na COVID-19 que, associados as citocinas liberadas durante essa doença aumentam a concentração de ferro intracelular e seu vazamento na circulação sanguínea. As altas concentrações de ferro liberam espécies reativas de oxigênio enquanto danificam tecidos próximos, mostrando-se como considerável fator de risco para o desenvolvimento da mucormicose ${ }^{6}$.

Pacientes infectados pelo SARS-CoV-2 recebem doses altas de esteroides sistêmicos, pois estes reduzem a inflamação nos pulmões e minimizam os danos provocados pela tempestade de citocinas. Entretanto, os pacientes afetados com a nova cepa da COVID-19 além de ser tratados com elevadas doses de corticosteroides, há o uso a longo tempo de máscaras de oxigênio e ventiladores tornando esses pacientes mais propensos à mucormicose. Além do mais, o uso de esteroides sistêmicos pode promover alterações na liberação de açúcar, o que contribui para o crescimento do Mucorales ${ }^{7}$.

Com base nos relatos de casos publicados, foi proposto uma relação entre mucormicose e pacientes positivos para COVID-19 como demonstrado na figura 1: 
Figura1: Relação entre pacientes positivos para COVID-19 e mucormicose

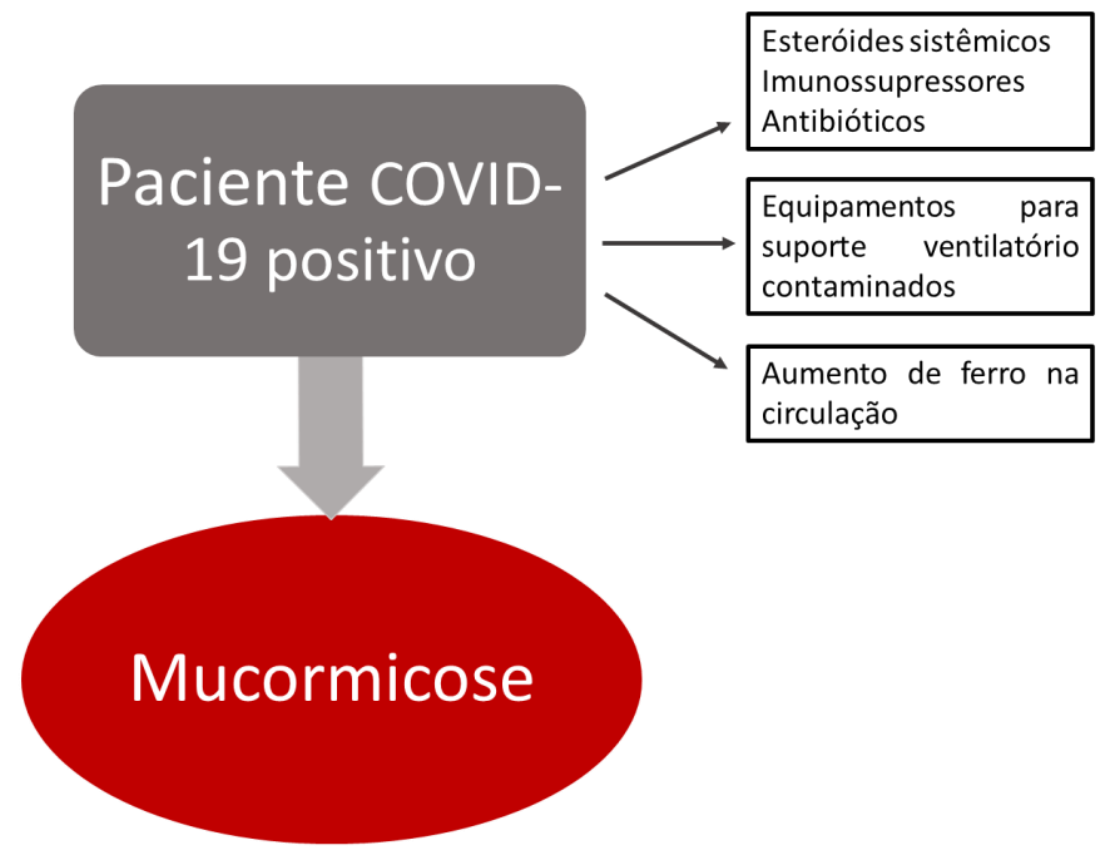

Fonte: adaptado de Mahalaxmi et al., 2021.

Diante dos casos de pacientes com COVID-19 apresentar infecções sistêmicas, para auxiliar no diagnóstico e diferenciação do possível agente fúngico associado, a ANVISA ${ }^{1}$ lançou uma nota técnica considerando que pacientes diabéticos descompensados com diagnóstico grave da COVID-19 que fez uso de corticoide e evoluiu durante ou após quadro de COVID-19 com quadro de sinusite aguda/subaguda deve seguir os seguintes critérios de avaliação, com imagem mostrando sinusite e ao menos UM dos sinais descritos na tabela 1:

Tabela 1: Sinais para auxiliar no diagnóstico de pacientes com COVID-19 associados à mucormicose

\begin{tabular}{|l|l|}
\hline \multicolumn{1}{|c|}{ SINAIS } & TEMPO \\
\hline $\begin{array}{l}\text { Dor aguda e localizada (com irradiação para } \\
\text { o olho) }\end{array}$ & PRECOCE \\
$\begin{array}{l}\text { Febre } \\
\text { Dor facial intensa } \\
\text { Comprometimento do estado geral }\end{array}$ \\
\hline $\begin{array}{l}\text { Úlcera nasal com exsudato negro } \\
\text { Sangramento nasal } \\
\text { Edema de face } \\
\text { Assimetrias } \\
\text { Dor ocular } \\
\text { Ptose palpebral }\end{array}$ & \\
\hline
\end{tabular}




\section{Alterações visuais}

Amaurose

Congelamento de movimentos oculares

Hematomas

Necrose ao redor do nariz

\section{Fonte: ANVISA, 2021.}

Em casos de manifestação da forma pulmonar podem-se considerar alterações radiológicas nos pacientes com sinal do halo reverso na Tomografia computadorizada de tórax, derrame pleural e múltiplos nódulos pulmonares ${ }^{1}$.

O diagnóstico de mucormicose consiste no isolamento de qualquer agente da ordem Mucorales com coleta de biópsia de pacientes com lesões em palato, seios da face ou pele, para análise microscópica, cultura e exame histopatológico. A presença de hifas hialinas, não septadas, com ramificação em $90^{\circ}$ no tecido obtido de biopsia confirmam a mucormicose ${ }^{1}$.

Infecções podem ser extremamente desafiadoras, mesmo em estágio inicial, por isso o diagnóstico precoce pode salvar vidas. Pacientes positivos para mucormicose são tratados com injeções de anfotericina-B, porém podem induzir efeitos colaterais incluindo danos renais. Em casos menos graves, a endoscopia foi inserida na cavidade nasal para remover o fungo. Na severa propagação de infecções, o cirurgião removerá a parte infectada ${ }^{7}$.

Em relação às infecções fúngicas existem fatores predisponentes para sua proliferação e disseminação, como internação prolongada, uso abusivo de antibióticos, nutrição parenteral, neutropenia, dentre outros ${ }^{14}$. Pacientes internados em unidade de terapia intensiva e que necessitam de ventilação mecânica, ou com maior duração de internações hospitalares são suscetíveis a desenvolver co-infecções fúngicas, principalmente pacientes COVID-19 positivos em estágios médio e avançados da doença ${ }^{15}$.

As infecções fúngicas sistêmicas não fazem parte da lista nacional de doenças de notificação compulsória no Brasil. Assim como também não são objeto de vigilância epidemiológica de rotina, devido a isso não existem dados epidemiológicos concretos da ocorrência, magnitude e transcendência dessas afecções sistêmica em nível nacional ${ }^{1}$. 
A mucormicose é uma infecção fúngica com uma alta taxa de mortalidade (50\%).

Além disso, um crescente número de pacientes com COVID-19 vem desenvolvendo essa infecção ainda no hospital ou após a alta, o que gera impactos diretamente relacionados à demora no diagnóstico e tratamento adequado ${ }^{7}$.

Diante disso a tabela 2 traz a proposta de tratamento da mucormicose no Brasil de acordo com a nota técnica elaborada pela ANVISA $^{1}$ para os casos de infecções fúngicas e COVID-19.

Tabela 2: Protocolo estabelecido pelo Ministério da Saúde para o tratamento de mucormicose

INFECÇÃO FÚNGICA TRATAMENTO

\begin{tabular}{|c|c|}
\hline Mucormicose & 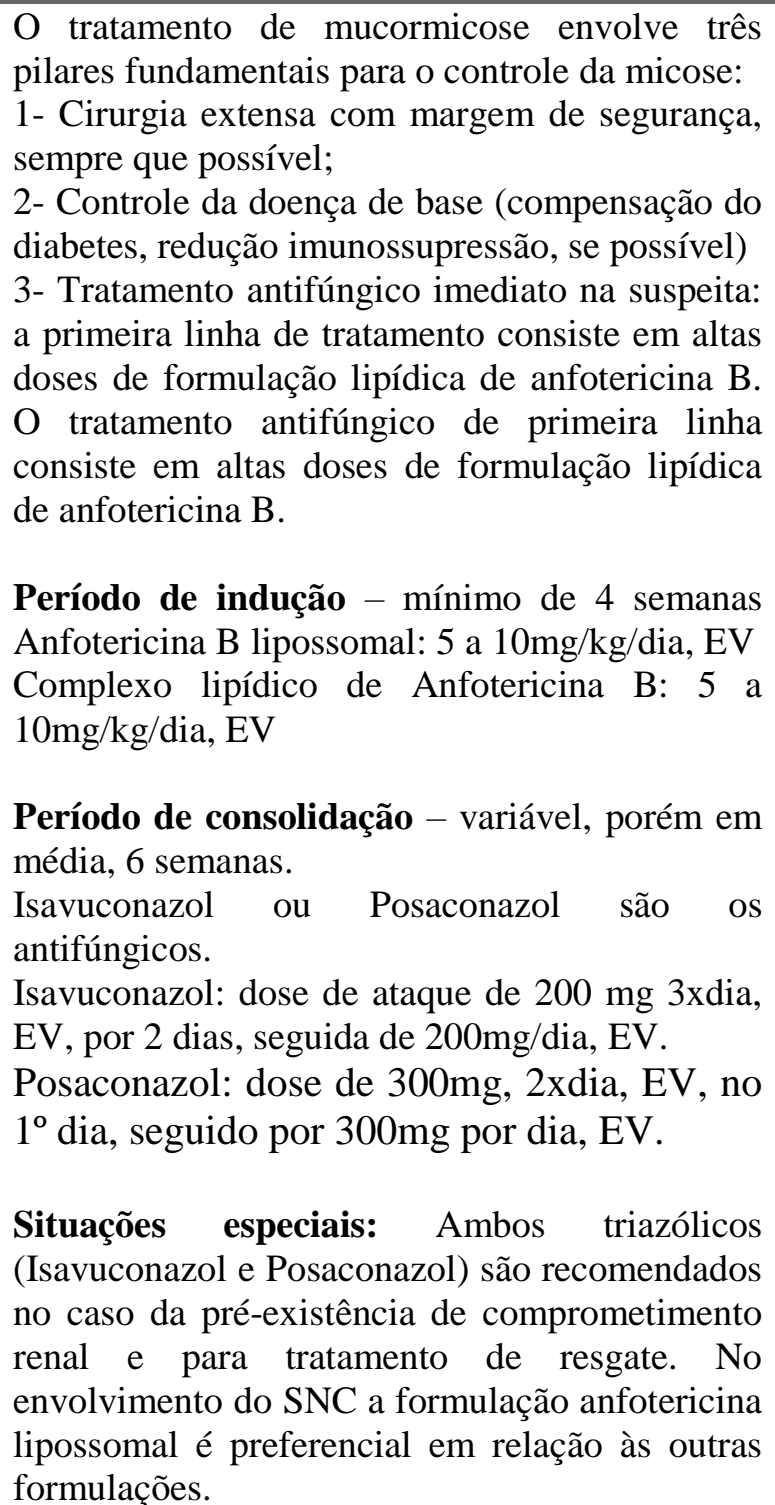 \\
\hline
\end{tabular}




\section{CONCLUSÃO}

A mucormicose é uma infecção fúngica oportunista emergente em pacientes afetados pela COVID-19. Trata-se de uma infecção fúngica com elevadas taxas de mortalidade por ser prevalente em pacientes imunodeprimidos, portadores de cetoacidose diabética, neutropênicos e que fizeram uso excessivo de esteroides sistêmicos. Frente à isso, há o desencadeamento da tempestade de citocinas que danificam órgãos celulares contribuindo para a gravidade da associação: COVID-19 e mucormicose.

Portanto é necessário que estratégias sejam elaboradas para reduzir a gravidade desses pacientes como: diagnóstico e terapia antifúngica precoces, debridamento cirúrgico, controle da doença de base (compensação do diabetes) e cautela com o uso de corticosteroides sistêmicos e antimicrobianos de amplo espectro. Priorizar a vacinação contra COVID-19 também é um dos principais fatores contribuintes para a redução da morbimortalidade durante a atual pandemia.

\section{REFERÊNCIAS}

1. Anvisa. Ministério da Saúde. Nota Técnica GVIMS/GGTES/ANVISA No 04/2021. Orientações para vigilância, identificação, prevenção e controle de infecções fúngicas invasivas em serviços de saúde no contexto da pandemia da COVID-19 - 14.06.2021. [acesso em 14 de Outubro de 2021]. Disponível em: https://www.saude.go.gov.br/files//conectasus/produtos-tecnicos/I\%20-\%202021/COVID-19\%20-

\%20Fungo\%20negro\%20(mucormicose).pdf

2. Pal R, Singh B, Bhadada SK, Banerjee M, Bhogal RS, Hage N, et al. COVID-19-associated mucormycosis: An updated systematic review of literature. Mycoses [Internet]. 2021;64(12):1452-9. Disponível em: http://dx.doi.org/10.1111/myc.13338 
3. Brasil. Ministério da Saúde. Mucormicose (Fungo Negro). [acesso em 14 de Outubro de 2021]Disponível em:

https://www.gov.br/saude/pt-br/assuntos/saude-de-a-a-z/m/mucormicose-fungo-negro

4. Ecdc - European Centre for Disease Prevention and Control. Communicable Disease Threats Report - CDTR Week 21, 23 - 29 May 2021. [acesso em 14 de Outubro de 2021]. Disponível em:

https://www.ecdc.europa.eu/sites/default/files/documents/Communicable-disease-threatsreport-29-may-2021.pdf

5. Monte Junior ES do, Santos MELD, Ribeiro IB, Luz G de O, Baba ER, Hirsch BS, et al. Rare and fatal gastrointestinal mucormycosis (zygomycosis) in a COVID-19 patient: A case report. Clin Endosc [Internet]. 2020;53(6):746-9. Disponível em: http://dx.doi.org/10.5946/ce.2020.180

6. John TM, Jacob CN, Kontoyiannis DP. When uncontrolled diabetes mellitus and severe COVID-19 converge: The perfect storm for mucormycosis. J Fungi (Basel) [Internet]. 2021;7(4):298. Disponível em: http://dx.doi.org/10.3390/jof7040298

7. Mahalaxmi I, Jayaramayya K, Venkatesan D, Subramaniam MD, Renu K, Vijayakumar P, et al. Mucormycosis: An opportunistic pathogen during COVID-19. Environ Res [Internet]. 2021;201(111643):111643. Disponível em: http://dx.doi.org/10.1016/j.envres.2021.111643

8. Ramphul K, Verma R, Kumar N, Ramphul Y, Mejias S, Lohana P. Rising concerns of Mucormycosis (Zygomycosis) among COVID-19 patients; an analysis and review based on case reports in literature. Acta Biomed [Internet]. 2021;92(4):e2021271. Disponível em: http://dx.doi.org/10.23750/abm.v92i4.11787

9. Hussain S, Baxi H, Riad A, Klugarová J, Pokorná A, Slezáková S, et al. COVID-19associated mucormycosis (CAM): An updated evidence mapping. Int J Environ Res Public 
http://dx.doi.org/10.3390/ijerph181910340

10. Slavin M, Thursky K. What is mucormycosis, the fungal infection affecting COVID patients in India? The Conversation. [acesso em 20 de Dezembro de 2021]. Disponível em: https://theconversation.com/what-is-mucormycosis-the-fungal-infection-affecting-covidpatients-in-india-160707.

11. Selarka L, Sharma S, Saini D, Sharma S, Batra A, Waghmare VT, et al. Mucormycosis and COVID-19: An epidemic within a pandemic in India. Mycoses [Internet]. 2021;64(10):1253-60. Disponível em: http://dx.doi.org/10.1111/myc.13353

12. Dilek A, Ozaras R, Ozkaya S, Sunbul M, Sen EI, Leblebicioglu H. COVID-19-associated mucormycosis: Case report and systematic review. Travel Med Infect Dis [Internet]. 2021;44(102148):102148.

em: https://www.sciencedirect.com/science/article/pii/S1477893921001897

13. Balachandar V, Mahalaxmi I, Subramaniam M, Kaavya J, Senthil Kumar N, Laldinmawii G, et al. Follow-up studies in COVID-19 recovered patients - is it mandatory? Sci Total Environ [Internet]. 2020;729(139021):139021. Disponível em: http://dx.doi.org/10.1016/j.scitotenv.2020.139021

14. da Silva BN, Portela FVM, de Mello Fiallos N, Aguiar ALR, de Aguiar Cordeiro R. Micoses oportunistas em pacientes neutropênicos. REMS [Internet]. 2020 [cited 2022 Jan 11];1(3):39-39. https://editoraime.com.br/revistas/index.php/rems/article/view/383

15. Song G, Liang G, Liu W. Fungal co-infections associated with global COVID-19 pandemic: A clinical and diagnostic perspective from China. Mycopathologia [Internet]. 2020;185(4):599-606. Disponível em: http://dx.doi.org/10.1007/s11046-020-00462-9 